\title{
First insights into Molecular basis Identification of 16s Ribosomal RNA Gene of Staphylococcus aureus Isolated from Sudan
}

Manal Abdalla Gumaa ( $\nabla$ therespect33@gmail.com )

University of Khartoum https://orcid.org/0000-0002-3580-3327

Abeer Babiker Idris

University of Khartoum

Nasr aldin Bilal Mohamed

University of Khartoum

Mohamed Ahmed Hassan

Detagen Genetic Diagnostics

Research note

Keywords: Staphylococcus aureus, 16S rRNA gene, phylogenetic tree

Posted Date: March 19th, 2021

DOI: https://doi.org/10.21203/rs.3.rs-322297/v1

License: (c) (i) This work is licensed under a Creative Commons Attribution 4.0 International License.

Read Full License 


\section{Abstract}

Objective: In this study, we analyzed the molecular evolution of Staphylococcus aureus isolates using 16S rRNA gene and phylogenetic analysis to detect the prevalence of $S$. aureus infections in Sudan.

Results: Molecular detection of S.aureus has shown that 20(43.47\%) of patients were positive for S.aureus. The phylogenetic tree of 16S rRNA sequences was divided into three lineages of S.aureus isolates detected from wound infections in Sudan. Nucleotides base-pair substitution was appeared at position 249. This mutation do not linked with Macrolides, Lincosamides and Streptogramines $b$ resistant phenotype. Further studies should investigate the effect of that mutation on resistance to other antibiotics.

\section{Introduction}

The increasing number of drug-resistant staphylococcal infections has created the need to investigating basic questions about how genetic variations that cause antibiotic resistance evolved within the population $[1,2]$. Consequently, In recent years, Sequence analysis of the $16 S$ rRNA gene is becoming more common as a genetic marker to confirm our understanding of S.aureus phylogeny and taxonomy and increasingly prevalent in the clinical environment $[3,4]$.

In fact, The geographic linkage within S.aureus is likely a result of interfamilial transmission incorporated with rearrangement within local communities[5, 6]. Few studies have been published in Sudan on $16 \mathrm{~S}$ rRNA gene sequencing. As described by Hassan et al, who showed the significance of microbial identification and phylogenetic markers for Staphylococci species from Sudanese isolates used for taxonomy[7]. Prior research also been explored in Sudan by Merghani et al, suggests that PCR assay with primers targeted to the 16S rRNA gene sequence offered a useful method for the identification of bacteria to the species level and differentiated one species from others[8, 9]. However, these studies cannot be considered as conclusive because the results did not correspond to the results of polyphasic taxonomy, and they found the related species cannot always be distinguished from each other [10].

Therefore, another promising line of research would be to detect the presence of mutations in $16 \mathrm{~S}$ rRNA and investigate the conservation of S.aureus sequences. These points have never previously been addressed in Sudan and the information regarding the African population is limited. This paper addresses to understand more completely the key tenets about molecular analysis of the 16S rRNA gene and phylogeny approach of staphylococcus aureus strains isolated from Sudanese patients with wound infections [11].

\section{Materials And Methods}

\subsection{Clinical isolates}


The study was carried out at hospitals (Khartoum state, in Soba teaching hospital and Military hospital). The process was accomplished in Medical Laboratory College at Khartoum University, Department of Microbiology and Molecular Biology. The study population includes patients who attended to hospitals for wound infections or post-operative surgical site infections. Questionnaires were used for patients to get their socio-demographic data. Forty-six wound swabs were collected from patients; records/information was anonymized and de-identified prior to analysis.

\subsection{Bacterial Identification}

After the samples were received to the laboratory, they inoculated on Mannitol salt agar (Oxoid $\mathrm{CM} 0085 \mathrm{~B}$ ), each isolated strains of Gram positive cocci were sub cultured on nutrient agar (Oxoid CM0 003B) and incubated at $37^{\circ} \mathrm{C}$ over night for biochemical reactions. S.aureus were identified by fermentation of mannitol, colonial morphology, Gram stain, Catalase test and coagulase test using conventional methods[12].

\subsection{Molecular Characterization}

\subsubsection{PCR Amplification of $16 \mathrm{~S}$ rRNA Gene}

The genomic DNA of S.aureus isolates were extracted from nutrient agar plates by guanidine chloride method as described previously by Alsadig et al., [13]. Then, PCR was carried out using universal oligonucleotide primers: 27F (5'-AGAGTTTGATCCTGGCTCAG-3') And 1492R (5' TACGGTTACCTTGTTACGACTT-3') [14]. The reaction mixture was included $1 \mu$ l of bacterial DNA, $22 \mu \mathrm{l}$ of $\delta \mathrm{dH} 2 \mathrm{O}$, and $\mathrm{I} \mu \mathrm{l}$ each primer in a final reaction volume of $25 \mu \mathrm{l}$. This mixture was added to the PCR master mix (GoTaq, Promega, USA) following the manufacturing guide. Then, run with a thermal cycler (SensoQuest, Germany) as follows: 30 cycles were performed in a thermocycler, each cycle has three steps of denaturation $\left(95^{\circ} \mathrm{C}\right.$ for $\left.1 \mathrm{~min}\right)$, annealing $\left(54^{\circ} \mathrm{C}\right.$ for $1 \mathrm{~min}$ ), extension $\left(72^{\circ} \mathrm{C}\right.$ for $3 \mathrm{~min}$ ) and final extension time of $72^{\circ} \mathrm{C}$ for 5 min [7]. Amplified products were analyzed by conventional electrophoresis, Bands were determined using an Imagemaster VDS image analysis system (SCIE- PIAS VISION U.K) [15]. The Sizes of the amplified products using universal primer were $1500 \mathrm{bp}$ which suggesting that bands is 16srRNA gene.

\subsubsection{Sequencing of $16 \mathrm{~S}$ rRNA gene}

DNA purification and Standard Sanger sequencing was conducted for ten isolates which were packaged according to the National Health Research Ethics Committee authorization and following the instructions of the sequencing company (Macrogen Inc. Seoul, Korea). The sequences was submitted in NCBI: https: // www.ncbi.nlm.nih.gov, with the accession numbers: from MT154222 to MT154231.

\subsubsection{Bioinformatic Analysis}

Nucleotide sequence isolates were visualized using Finch TV program (version 1.4.0)[16]. In order to search for nucleotide sequences similarity, Genbank databases were used by online program nucleotide 
BLAST (http://www.ncbi.nlm.nih.gov/blast/Blast.cgi)[17]. Closely related sequences were explored from $\mathrm{NCBI}$ and subjected to multiple sequence alignment by BioEdit program (version 7.2).[18, 19]. Gblocks was used to estimate the quality of each sequence, edit and eliminate poor quality sequences[20]. The Neighbor-Joining phylogenetic tree was carried out by MEGA software using default parameters (http://www.megasoftware.net/index.html)[21][22, 23].

\section{Results}

\subsection{Analysis of 16S rRNA sequences}

10 sequences of 16 S rRNA of S. aureus from Sudanese patients were characterized by PCR to investigate the mutations and their conservative nature; Products band had a clear chromatogram. Sequence analysis by BLASTn revealed similarity with few differences with S.aureus from Japan (LC508802), China (MN923027), Pakistan (MN611106), Nigeria (MN606199), Bangladesh (MN611246), Egypt (MN556575), Iraq (MN555444) and China (MN652637) as shown in Table S1, Supplementary Material File. Regarding mutations, the alignment of our isolated sequences showed that tow isolates (21 and 38) exhibited basepair substitution was appeared at position 249 from A to G, Figure S1 (A), Supplementary Material File. Multiple sequence alignment of the isolates with S.aureus Genbank strains confirmed the presence of that variation from isolates and from selected published nucleotide sequences compared with the reference strain, Figure: S1(B), Supplementary Material file.

\subsection{Phylogenetic tree structuring (Maximum likelihood tree)}

The cladogram graphic of a phylogenetic tree diverged into three lineages. All the Sudanese S.aureus strains clustered with strains from different countries. The 16S rRNA sequence of strains 7 and 38, although clustered with other global strains in one clade, had a novel $A \rightarrow G$ substitution at nucleotide position 249 as a kind of strain evolution. However, mutant strains 7 and 38 shared a common ancestor with strains from Japan (LC508802), Nigeria (MN606199), Germany (MF664194) and Italy (MN811085); and represented with them a separated clade with a bootstrap value of $99 \%$ as shown in Figure S2, Supplementary Material file.

\section{Discussion}

The reports found a novel strain of the 16S rRNA gene in tow isolates had not been previously reported. They shared a single nucleotide change from $A$ to $G$ distinguished them from the consensus sequence as a type of strain evolution.

The Situation of mutant isolates in separated branches makes it a most recent common ancestor of those groups. And revealed that the possible source of our mutant isolate is Ethiopia; this may be due that Ethiopia is the nearest country to Sudan among these countries. This agrees with similar outcomes found $16 \mathrm{~S}$ rRNA sequencing can used to identify genetically atypical bacterial isolates from different sources $[15,24,25]$. 
The phylogenetic tree also exhibited different lineages of S.aureus strains detected from several hospitals in Sudan which indicated differential

evolution. The isolates distributed in the three branches with those from Japan (LC508802), Nigeria (MN606199), Germany (MF664194), Italy (MN811085), Ethiopia (MK217496), Iraq (MN555444), Pakistan (MN611106), Bangladesh (MN611246), Egypt (MN556575), China (MN652637), India (MK165143) and USA (MF385261). The current results agree with previouly reported study in Sudan by Mohamed et a/ that found genetic similarity in genomic sequence analyses of different S.aureus strains in the world in relation with isolated strains [26]. Similar to the current study results, Raed et al indicated that genetic dimension between Iraq and the isolates of the world is extremely relative, and 16s rRNA analysis is considered a good discrimination approach for distinguishing unrelated isolates [27].

Macrolides, Lincosamides and Streptogramines antibiotics grouped into a single family because they share a similar binding site in subunit ( $23 \mathrm{~s}$ rRNA) of the bacterial ribosome. Alteration of a specific base of rRNA reduced drug affinity and evokes the resistance (MLSB phenotype) [28-30]. Phenotypic expression of MLSB resistance in staphylococci can either inducible or constitutive [30].

From the short review above, we showed that the 12 (75\%) iMLSB phenotype isolates prevailed over the 4 (25\%) CMLSB phenotype which is slightly vary from other study performed in Sudan by Mahmoud et al which they found (25.4\%) S.aureus isolates yielded inducible resistance[31]. different results performed in Sudan by Makarem et al found all S.aureus isolates were sensitive to erythromycin antibiotic[32]. It appears that there was no association between the occurrence of the resistant in MLSb S.aureus and 16S rRNA gene mutations. Overall this findings was in accordance with pervious findings found the amount of Methylated adenine in 16S rRNA is not affected by erythromycin[33].

\section{Conclusion}

The phylogenetic analysis of 16S rRNA sequences identified several lineages of S.aureus isolates detected from wound infections in Sudan. The mutation which discovered in 16S rRNA region do not associated with Macrolides, Lincosamides and Streptogramines b resistant phenotype. The application future direction of this paper is using DNA sequencing and insilico analysis depending on 16S rRNA and phylogeny approach to differentiate between closely related strains and study taxonomy relationships between bacteria. Future research should consider the potential effects of that mutation on resistance to other antibiotics.

\section{Limitations}

However, the approach utilized suffers from the limitation that based on small sample size. An extra search is needed to highlight the variation which may occur and cover prevalent strains in different geographic regions in Sudan to obtain more complicated evolutionary events. 


\section{Declarations}

\section{Ethics approval and consent to participate}

The study protocol was approved by the National Health Research Ethics Committee authorization and University of Khartoum. The patients gave informed written consent before they enrolled in the study.

\section{Consent for publication}

The patients gave written informed consent before they registered in the study.

\section{Availability of data and material}

The data supporting our findings can be found in Medical Laboratory College at Khartoum University, Department of Microbiology.

\section{Competing interests}

The authors declare that there are no conflicts of interest.

\section{Funding Statement}

The authors received no specific funding for this work.

\section{Author's contributions}

Not applicable

\section{Acknowledgements}

The authors kindly thank the patients who participated in the study and the staff of Soba teaching hospital and Military hospital for their support in sample collection. And also we acknowledge Dr. Muzamil Abdel Hamid from institute of endemic diseases for his support.

\section{References}

1. Schaechter M, Desk encyclopedia of microbiology. 2010: Academic Press.

2. Petit III, R.A. and Read TDJP, Staphylococcus aureus viewed from the perspective of 40,000+ genomes. 2018. 6: p. e5261.

3. Kawasaki S, et al., Species-specific identification of campylobacters by PCR-restriction fragment length polymorphism and PCR targeting of the gyrase B gene. 2008. 74(8): p. 2529-2533.

4. Janda JM. and S.L.J.J.o.c.m. Abbott, 16 S rRNA gene sequencing for bacterial identification in the diagnostic laboratory: pluses, perils, and pitfalls. 2007. 45(9): p. 2761-2764. 
5. Mamoun M, S.J.A.J.o.M R, Elsanousi. Molecular Identification of 16s Ribosomal RNA Gene of Helicobacter pylori Isolated from Gastric Biopsies in. Sudan. 2015;3(2):50-4.

6. Szymanek-Majchrzak K, et al. Effect of Selective Antibiotic Pressure on the MLS-B Phenotype in Methicillin-Resistant Staphylococcus aureus Strains Originating From Patients From Transplantation Wards: 24 Years of Observations. in Transplantation proceedings. 2018. Elsevier.

7. Alfatih YM, et al., Detection of a novel mutation G511T in the 530 loop in 16S rRNA in multi drugs resistant Mycobacterium tuberculosis isolated from Sudanese patients. 2018: p. 497628.

8. Merghani EAE, et al., Identification of Bacterial Pathogens Isolated from Haemodialysis Patients using VITEK 2 Compact System and their Antibiotic Susceptibility. 2016.

9. Coutinho VdLS, et al., Distribution of erm genes and low prevalence of inducible resistance to clindamycin among staphylococci isolates. 2010. 14(6): p. 564-568.

10. Mamoun, MJ.A.J.o.MR. Molecular Identification of 16s Ribosomal RNA Gene of Helicobacter pylori Isolated from Gastric Biopsies in. Sudan. 2015;3(2):50-4.

11. Ayeni FA, B.T.J.T.J.o.P R, Odumosu. False identification of other microorganisms as Staphylococcus aureus in Southern Nigeria. 2016;15(9):1941-5.

12. MacFaddin JF, Pruebas bioquímicas para la identificación de bacterias de importancia clínica. 2003: Ed. Médica Panamericana.

13. Gassoum A, et al. Allele Frequency Of P53 Gene Arg72Pro In. Sudanese Meningioma Patients Controls. 2014;6(3):243-8.

14. Liu W, et al., Analysis of microbial composition in acid whey for dairy fan making in Yunnan by conventional method and 16S rRNA sequencing. 2009. 59(2): p. 199-205.

15. Idris AB, et al., Molecular Phylogenetic Analysis of 16S rRNA Sequences Identified Two lineages of $H$. pylori Strains Detected from Different Regions in Sudan Suggestive of Differential Evolution. 2019.

16. Ozdilek A, et al., Molecular phylogeny of relict-endemic Liquidambar orientalis Mill based on sequence diversity of the chloroplast-encoded matK gene. 2012. 298(2): p. 337-349.

17. Donkor ES, et al., Bioinformatics with basic local alignment search tool (BLAST) and fast alignment (FASTA). 2014. 6(1): p. 1-6.

18. Hall T, Biosciences I, Carlsbad CJGBB. BioEdit: an important software for molecular biology. 2011;2(1):60-1.

19. McWilliam H, et al., Analysis tool web services from the EMBL-EBI. 2013. 41(W1): p. W597-W600.

20. Castresana JJMb, evolution. Selection of conserved blocks from multiple alignments for their use in phylogenetic analysis. 2000;17(4):540-52.

21. Kumar S, et al., MEGA7: molecular evolutionary genetics analysis version 7.0 for bigger datasets. 2016. 33(7): p. 1870-1874.

22. Osman NAM, et al. Molecular study of Panton-Valentine Leukocidin genes among Staphylococcus aureus clinical isolates in Khartoum State. Sudan. 2015;3(3):107-11. 
23. Stecher G, Tamura K, Kumar S, Molecular Evolutionary Genetics Analysis (MEGA) for macOS. Molecular Biology and Evolution, 2020.

24. Zakerbostanabad S. Molecular characterization of Staphylococcus aureus Isolated from Clinical Samples Based on 16srRNA, rpoB and hsp70 Genes by MLSA. Journal of Infection and Public Health, 2019.

25. Matuszewska M, et al. The Evolutionary Genomics of Host Specificity in Staphylococcus aureus. Trends Microbiol. 2020;28(6):465-77.

26. Ali MS, et al., Genomic analysis of methicillin-resistant Staphylococcus aureus strain SO-1977 from Sudan. 2019. 19(1): p. 126.

27. Al-Obaidi MMJ, Suhaili Z. and M.N.M.J.E.b.I.A. Desa, Genotyping Approaches for Identification and Characterization of Staphylococcus aureus. 2018: p. 85.

28. Bailey AM, et al., RamA, a member of the AraC/XylS family, influences both virulence and efflux in Salmonella enterica serovar Typhimurium. 2010. 192(6): p. 1607-1616.

29. Igrejas G, et al., Surveying Antimicrobial Resistance: Approaches, Issues, and Challenges to Overcome. 2017: Frontiers Media SA.

30. Mayers DL, et al., Antimicrobial Drug Resistance: Mechanisms of Drug Resistance. Vol. 1. 2017: Springer.

31. Mahmoud AM, et al., Inducible clindamycin resistance and nasal carriage rates of Staphylococcus aureus among healthcare workers and community members. 2015. 15(3): p. 861-867.

32. Salih MA, Abdalla AM, Masri MAR. Detection of Erythromycin Resistance Genes erm (A), erm (B), erm (C) and msr (A) in Staphylococcus Nasal carriers in Khartoum State.

33. Lai C. and B.J.P.o.t.N.A.o.S. Weisblum, Altered methylation of ribosomal RNA in an erythromycinresistant strain of Staphylococcus aureus. 1971. 68(4): p. 856-860.

\section{Supplementary Files}

This is a list of supplementary files associated with this preprint. Click to download.

- SupplementaryMaterial.docx 